\title{
A Comprehensive Update of the Treatment and Management of Bertolotti's Syndrome: A Best Practices Review
}


F. Sherman, MD, MBA ${ }^{4}$, Antonella Paladini, MD, PhD ${ }^{5}$, Giustino Varrassi, MD, PhD, FIPP ${ }^{6}$, Amira S. Odisho, MD, Sumitra Miriyala, $\mathrm{PhD}, \mathrm{MBA}^{8}$, Alan D. Kaye, MD, $\mathrm{PhD}^{9}$

${ }^{1}$ School of Medicine, Georgetown University, ${ }^{2}$ Department of Anesthesiology, Critical Care, and Pain Medicine, Beth Israel Deaconess Medical Center, Harvard Medical School, ${ }^{3}$ Department of Anesthesiology, Mount Sinai Medical Center, ${ }^{4}$ Department of Orthopaedic Surgery, Tulane University, ${ }^{5}$ Department MESVA, University of L'Aquila, ${ }^{6}$ Via Tacito 7, Paolo Procacci Foundation, ${ }^{7}$ Department of Psychiatry and Behavioral Medicine, LSUHSC School of Medicine, ${ }^{8}$ Department of Cellular Biology and Anatomy, LSUHSC School of Medicine, ${ }^{9}$ Department of Anesthesiology, LSUHSC School of Medicine

Keywords: steroid injection, local anesthetic, epidural injections, radiofrequency ablation, spine fusion, transitional vertebra, lower back pain, bertolotti's syndrome

https://doi.org/10.52965/001c.24980

\section{Orthopedic Reviews}

Vol. 13, Issue 1, 2021

Bertolotti's Syndrome is defined as chronic back pain caused by transitional lumbosacral vertebra. The transitional vertebra may present with numerous clinical manifestations leading to a myriad of associated pain types. The most common is pain in the sacroiliac joint, groin, and hip region and may or may not be associated with radiculopathy. Diagnosis is made through a combination of clinical presentations and imaging studies and falls into one of four types. The incidence of transitional vertebra has a reported incidence between 4 and 36\%; however, Bertolotti's Syndrome is only diagnosed when the cause of pain is attributed to this transitional anatomy. Therefore, the actual incidence is difficult to determine.

Initial management with conservative treatment includes medical management and physical therapy. Injection therapy has been established as an effective second line. Epidural steroid injection at the level of the transitional articulation is effective, with either local anesthetics alone or in combination with steroids. Surgery carries higher risks and is reserved for patients failing previous lines of treatment. Options include surgical removal of the transitional segment, decompression of stenosed foramina, and spinal fusion. Recent evidence suggests that radiofrequency ablation (RFA) around the transitional segment may also provide relief.

This manuscript is a comprehensive review of the literature related to Bertolotti's Syndrome. It describes the background, including epidemiology, pathophysiology, and etiology of the Syndrome, and presents the best evidence available regarding management options.

Bertolotti's Syndrome is considered an uncommon cause of chronic back pain, though the actual incidence is unclear. Most evidence supporting these therapies is of lower-level evidence with small cohorts, and more extensive studies are required to provide strong evidence supporting best practices.

\section{INTRODUCTION}

Bertolotti's Syndrome is defined as chronic low back pain caused by lumbosacral transitional vertebrae. In the spines of these patients, the enlargement of the caudal lumbar vertebrae at the transverse process leads to the fusion or articulation of the transverse process with the sacrum or the ilium, causing discogenic disease and limiting mobility. ${ }^{1}$

\footnotetext{
a Corresponding Author: Hisham Kassem Mount Sinai Medical Center Department of Anesthesiology 4300 Alton Road Miami Beach, FL 33140 Phone: (305) 987-8008 hkassem.md@gmail.com
} 
This congenital lumbosacral transitional vertebra (LSTV) defect, which has various presentations, may present as lower back pain syndrome in the third and fourth decade of a patient's life. Once the cause of the low back pain has been established as LSTV, it is classified as Bertolotti's Syndrome. ${ }^{2}$ The pain caused by Bertolotti's Syndrome is not uniform and originates from different pathology caused by the LSTV. These pathologies include scoliosis, arthropathy of the joints, and muscle strains of the quadratus lumborum and iliopsoas. ${ }^{1}$ The deformation of the transitional vertebra may cause nerve compression due to compressed discs resulting in neuropathic pain. ${ }^{1}$ On average, patients report daily pain at above $5 / 10 .{ }^{3}$ Along with significant pain, patients with Bertolotti's Syndrome self-report an average of over $36 \%$ on the Oswestry disability scale, correlating to a moderate disability that affects many aspects of daily life. ${ }^{3-5}$ The patients who have LSTV tend to have a more significant spinal degeneration above the deformity than other conditions leading to chronic lower back pain. However, they tend to have similar levels of pain and disability to those that have back pain without having LSTV. ${ }^{3}$

Related to its similarity in pain and disability with other causes of back pain, Bertolotti's Syndrome is diagnosed in two parts; a clinical assessment of pain and advanced imaging demonstrating these anomalies. ${ }^{1-6}$ Clinically, Bertolotti's Syndrome presents various pain and tenderness, symmetrically or asymmetrically, at the sacroiliac area, hip, and groin. There may be radicular pain due to nerve compression or pseudo-radicular pain-related due to the bone-on-bone contact at the pseudo-articulate joint of the transverse vertebra. ${ }^{2-7}$ A clinical exam can include an intra-articular diagnostic block with lidocaine $2 \%$ and bupivacaine $0.5 \%$ at either the sacroiliac joint or the facet joint under fluoroscopic guidance. Achieving an $80 \%$ decrease in pain following these injections is diagnostic. ${ }^{1}$ Bertolotti's Syndrome presents with non-specific back pain; therefore, confirmation of the diagnoses must be made through radiologic examination of the lumbosacral spine. ${ }^{1}$ The lumbosacral spine radiographs will demonstrate unilateral or bilateral enlargement of the transverse processes with potential articulation with the sacrum or ilium. ${ }^{8}$ These can be complemented with an MRI of the lumbosacral spine if there is radicular pain leading to a suspicion of discogenic pain. ${ }^{1}$

Bertolotti's Syndrome encompasses many types of LSTV that are defined under the Castellvi system. In 1984, Dr. Antonio Castellvi determined four defining types of LSTV through examinations of myelograms of 200 patients. ${ }^{9}$ These four types of LSTV include: Type I-dysplastic transverse process that is at least $19 \mathrm{~cm}$ wide, Type II-transverse process growth leading to an incomplete sacralization or lumbarization of the transverse process, Type III-complete transverse process sacralization/lumbarization, and Type IV- mixed complete sacralization and incomplete sacralization. ${ }^{9}$ Of the Castellvi classifications, the most commonly seen LSTV is Type 1 (42\%), followed by Type II (38\%), Type III (8\%), and Type IV (5\%). ${ }^{9}$ Disc herniations are often present at single levels, although some patients have herniations of multiple discs due to the lumbosacral transitional vertebra. 9

\section{PATHOPHYSIOLOGY}

The presence of an LSTV causes changes in the biomechanics of the spine. Normal skeletal anatomy utilizes the sacrum's size, surface area, and sacroiliac joint to dissipate the upper body's weight across the fused bony sacrum and into the legs. ${ }^{10}$ However, the presence of an LSTV has been shown to cause hypomobility at the L5/S1 level and hypermobility at the superjacent and superior lumbar levels. ${ }^{11}$

For over a century, the disruption in the biomechanical axis of the spine caused by LSTV has been argued to be causally related to the development of lower back pain. The development of lower back pain is hypothesized to be from the earlier presentation of arthritic changes, disc herniation, disc degeneration, or spinal canal and foraminal stenosis. ${ }^{12,13}$ Otani et al. found in 2001 that disc herniations occurred more frequently (17\% vs. $11 \%)$ and at an earlier age (35 yo vs. 59 yo) in patients with LSTV than those without LSTV. ${ }^{14}$ Additionally, studies have shown that foraminal stenosis, disc degeneration, and facet degeneration occur more frequently at the level immediately above the LSTV than at that same level in patients without LSTV. ${ }^{15}$

Hanhivaara et al. found that LSTV of Castellvi Types II, III, and IV are associated with higher amounts of lumbar disc degeneration at all lumbar levels except L2-L3. ${ }^{16}$ In the setting of acute lumbar disc herniation, Kanna et al. demonstrated the presence of an LSTV was the most significant predictor of a failed selective nerve root block with an Odds Ratio of 20.1. ${ }^{17}$ In patients with low back pain, the presence of an LSTV is linked with a significantly higher amount of sacroiliac joint dysfunction than in those without an LSTV. ${ }^{18}$

\section{EPIDEMIOLOGY}

Bertolotti's Syndrome has a widely debated incidence. Studies have projected that between 4 and $36 \%$ of people have LSTV. ${ }^{19}$ Hsieh et al. analyzed patients who had no current symptoms and determined by radiographic imaging that $4 \%$ of the population of chiropractic patients had LSTV of Castellvi types II-IV. ${ }^{20}$ Examining radiographs of 6200 patients in the general population, Ucar et al. determined that $18.7 \%$ of people in the general public have LSTV. ${ }^{21}$ The study determined that there are significantly more men with LSTV than there were women with LSTV in the general population. ${ }^{21}$ As well, there are significantly more men with sacralization of their LSTV than women. ${ }^{21}$ This study found that the Castellvi Type Ia and Ib dominated the diagnosis of LSTV followed in order by IIa and IIb, IIIa and IIIb, and IV. ${ }^{21}$ Although there is a high incidence of LSTV in the general population, many are asymptomatic, leading to a debate about whether LSTV is a true cause of back pain. While Castellvi determined pain is derived from the anomaly of the lumbar spine, others have determined pain and severity of back pain are not associated with LSTV. ${ }^{9,14,22}$ Due to the historical uncertainty of the connection between lower back pain and the LSTV, it is difficult to define the true incidence of patients with Bertolotti's Syndrome. To determine its actual prevalence, Elster examined 2000 patients who lived 
with lower back pain and determined that 140 (7\%) patients had LSTV and thus were diagnosed with Bertolotti's Syndrome. ${ }^{23}$

\section{CONSERVATIVE MANAGEMENT}

Case reports have demonstrated a successful reduction in pain through a 1-month follow-up using an injection of triamcinolone acetate and lidocaine at the articulation of the ilium and the transverse process. ${ }^{24}$ Physical therapy represents another modality, with one case report utilizing lumbosacral manipulation and exercises resulting in improvements in pain and mobility over two weeks. ${ }^{25}$ Similarly, two patients undergoing manual therapy under the care of a chiropractor noted substantial but incomplete improvement, one at two weeks and one at four weeks, in their perceived pain levels and mobility. ${ }^{26}$ While not explicitly addressed in Bertolotti's Syndrome, a 2015 systematic review reported Pilates may provide short-term benefit over minimal physical exercise or no intervention for patients with non-specific chronic low back pain. ${ }^{27}$ Authors did note large heterogeneity in the analyzed studies, including outcome measures and actual Pilates protocols. ${ }^{27}$ As much of the current data is based on small populations with highly variable follow-up, further study is needed before reliable conclusions on conventional management can be drawn.

Forms of conservative management for Bertolotti's Syndrome include activity modification, pharmacologic therapy, physical therapy, and interventional therapy. ${ }^{28}$ In a case series of 5 patients with heterogeneous symptoms and pain generators, Zhang et al. suggested that transforaminal or interlaminar epidural steroid injections may provide some benefit when presentations are consistent with irritation of the nerve root. ${ }^{28} \mathrm{~A}$ recent review by Holm et al. highlights the difficulty of directly comparing the current data on steroid management as compared to surgery. ${ }^{29}$ In one prior study, the use of local lidocaine at the pathologic joint in 10 of 11 patients in the cohort led to 5 who were pain-free and 4 with immediate pain reduction. ${ }^{30}$ These patients were not naïve to injections or physiotherapy, and all 11 were eventually given surgical management. ${ }^{30}$ The outcomes at follow-up were improved or pain-free in 9 of 11 , and the authors hypothesized that response to anesthetic injection might correlate to future successful surgical management. 30

In contrast, Santavirta et al. did not necessarily note this association. ${ }^{31}$ Of 16 study patients for surgical intervention, 6 had local anesthetic injections in the transitional articulation, which authors noted did transiently relieve the pain. ${ }^{31}$ However, only 4 of the 6 later had a successful surgical intervention, leading to the conclusion that a preoperative response may not correlate well with the ultimate treatment result; however, they still did recommend a role in using anesthetic injections in the diagnostic workup. ${ }^{31}$ Additionally, as local anesthetic and steroids are sometimes given within a short period, or perhaps even mixed, it makes isolating the effects of steroids themselves difficult. In a study of 15 patients comparing injections of lidocaine with saline versus lidocaine with cortisone (glucocorticoid receptor agonist), for symptomatic LSTV type II or IV, there were no differences between groups, yet when pooled pa- tients did experience significant reductions in mean VAS scores from baseline to 4 weeks as well as the 12 -week timepoint. ${ }^{32}$ Combined injections serve as a common approach, as Marks et al. reported 8 of 10 patients in the cohort studied experienced total relief within 30 minutes of infiltration with methylprednisolone acetate and lidocaine with relative pain relief lasting from 1 day to over 27 months in this cohort. ${ }^{33}$

\section{SURGICAL MANAGEMENT}

There are surgical options to treat Bertolotti's Syndrome. Although an effective method of treatment when indicated, there are inherent risks with surgery. One of the most common forms of treatment is resection, also known as the "Processectomy," of the lumbosacral transitional vertebra. This surgery addresses back pain in a patient with LSTV related to the mechanical stress of the pseudo articulation or the articulation. ${ }^{30,34,35}$ To determine if the pain is from the LSTV, a diagnostic anesthetic block is done, looking for positive pain relief. ${ }^{34}$ If positive, a surgeon may partially remove or completely resect the LSTV. ${ }^{35}$ Studies reviewing the outcomes of the surgical resection of the LSTV have demonstrated positive outcomes in these cohorts. In a study by Jonsson et al. following eleven patients undergoing resection, seven patients had alleviated all of their pain, and two of the patients had alleviated a proportion of their pain. ${ }^{30}$ Four of the patients reported increased mobility directly following the resection due to the freed transverse process and decreased biomechanical stress. ${ }^{30}$ of the two failed surgeries, one was determined to be due to previous degeneration of the spine, while the failure of the other was due to unknown factors. ${ }^{30}$ As such, resection of the LSTV should be performed in younger patients who do not present with degeneration of the spine. ${ }^{30,31}$

In a retrospective study, patients with Bertolotti's Syndrome of Castellvi type I or II who underwent the transverse processectomy had decreased pain, as reported by the VAS pain quantifying system, of $50 \%$ from their preoperative levels. ${ }^{34,36}$ Despite the relief in the pain levels that the patients had, five of the eight patients who had undergone the resection presented with degeneration of the disc above the previously resected vertebrae. Three of those who had the resection were subjected to a second surgery due to the failure of the first surgery to manage the symptoms. ${ }^{31}$ Resection should be limited to select patients; those absent of adjacent transitional vertebrae disc pathology are most likely candidates for the resection procedure. ${ }^{31}$

A spine fusion is a surgical procedure that helps relieve the chronic pain caused by Bertolotti's Syndrome. A study by Santavirta followed eight patients who underwent a resection of the transverse process and eight who underwent a posterolateral fusion. When comparing the long-term outcomes, the fusion cohort had an increased disc degeneration in the disc immediately superior to the fusion, with 7 of 8 patients showing degeneration at follow-up. ${ }^{31}$ However, patients with previous disc degeneration of the LSTV disc, but not the superior disc, fared better with the fusion than the resection. ${ }^{31}$ As such, it is recommended that fusion be considered on selected patients who have previous disc pathology. 
In examining surgical efficacy compared to non-operative management, Santavirta demonstrated the surgical group fared only slightly better than the non-operative controls (1.9 versus 2.5 on the Oswestry Scale), independent of which surgery they received. 31,37 Undergoing a surgical procedure is considered a last line of treatment. However, it can manage pain effectively, as seen in the studies of patients receiving resections and fusions. Although these results were positive, it is recommended that surgery, whether resection or fusion, not be the first line of treatment for patients with Bertolotti's Syndrome. Still, instead, patients should be offered physiotherapy, ergonomic relief efforts, and other less invasive treatment options before being offered the opportunity for surgery. ${ }^{30,31,35}$

Some patients undergo decompression of the stenosed foramen below the LSTV. Patients who have radiculopathy due to the LSTV are candidates for nerve decompression surgery. The decompression requires the translocation of the nerve medially and the resection of the bone spur or pseudoarticulation. ${ }^{38}$ A recorded complication from this surgery was transient hypesthesia that resolved after two weeks. ${ }^{38}$ Certain hypothesized positioning variations to the surgical methods have been proposed to minimize the temporary hypesthesias of the leg. ${ }^{38}$ A study by il Ju et al. examined decompression in conjunction with resection surgery, documenting a decrease in the VAS pain score by $72 \%$ postoperatively. ${ }^{34}$ Decompressive surgery of the nerve root can relieve pain by freeing the nerve from the anomalous growth of the transverse process. Related to its variation of anatomical presentation, surgeons must take an individualized approach to a surgical operation on patients with Bertolotti's Syndrome. ${ }^{7}$

\section{RADIOFREQUENCY ABLATION}

Continuous radiofrequency ablation (RFA) for pain management arose in the mid to late 20th century, designed to create coagulative necrosis using a high-frequency alternating current. ${ }^{39}$ Several types of nerve ablation exist, including chemical, direct electrical, cryoablation, and radiofrequency-based ablation, the latter of which spans subtypes such as pulsed RFA, cold RFA, and bipolar RFA. In considering an approach to general sacroiliac joint pain, unlike ablation for lumbar nerves, there is more anatomic variation in sacral branches. ${ }^{40}$ A cooled RFA technique was developed to generate a larger lesion to compensate for variable nerve locations. However, compared to traditional RFA, this technique did not lead to any difference through 12 months, with only about $40 \%$ of patients in both groups reporting over $50 \%$ pain relief at the 3 -month time point. ${ }^{41}$

A newer method under investigation involves bipolar RFA therapy along a strip overlying the sacrum targeting L5-S3. This newer method requires further study but may represent an improvement over cold-RFA, with over $70 \%$ of patients retaining over $50 \%$ pain relief at three months. ${ }^{42}$ The development of these ablative techniques in general sacroiliac joint pain may warrant their investigation in Bertolotti's Syndrome. In a report out of Alberta, Canada, a 56-year-old woman with initial pain treated with L4/L5 facet joint RFA did not have a durable response, was found to have an elongated L5 transverse process with sacral ar- ticulation, and was finally treated with bipolar RFA strip lesions around this area, leading to complete pain control until 16-month follow-up. ${ }^{43}$ Their initial protocol was to lesion circumferentially around the articulation, using fluoroscopic guidance and two 18-gauge RF needles placed $2-3 \mathrm{~mm}$ from the articular margins, using a temperature of 80 degrees Celsius and contact time of 90 seconds. ${ }^{43}$ This case report was one of the first to document a successful RFA technique for Bertolotti syndrome. ${ }^{43,44}$

More recently, a team from Japan reported success with pulsed RFA among four patients with Castellvi type IIa/ b through a mean follow-up at eight months, using pulse widths of $20 \mathrm{~ms}$ and $20 \mathrm{~V}$ at 42 degrees Celsius for 4 minutes at three sites. ${ }^{45}$ They reported that in their experience, this technique led to less procedural pain compared to thermal RF. ${ }^{45}$

A current limitation with the analysis of these case reports is the uncertainty of the pain generator and how management should differ in the various subtypes of LSTV. ${ }^{10,24}$ In a study restricted to 20 Bertolotti's syndrome Castellvi type 1a patients with scoliosis, the most common source of pain was the ipsilateral facet joint; however, contralateral facet joint pain, sacroiliitis, discogenic pain, and non-articulation pain were also reported. ${ }^{1}$ We were unable to find specific applications of discogenic therapies for Bertolotti's Syndrome; however, the following two examples represent possible future directions based on work in general lower back pain patients. If the patient's pain is discogenic in nature, two or more treatments of RF lesioning of the ramus communicans at 80 degrees Celsius for 60 seconds may improve such pain. ${ }^{46}$ Another approach for discogenic pain involves introducing a thermal resistive coil into the center of the target disc, heating the coil to 90 degrees Celsius, corresponding to an annular temperature in the 60-degree Celsius range, and leaving it in place for 4 minutes. This approach aims to shrink collagen and coagulate nerve tissue, thus theoretically addressing both mechanical and nociceptor components of discogenic pain and was reported to be efficacious in some LBP patients. ${ }^{47}$ Because patient presentation may be heterogeneous. The most successful therapy is hard to predict initially. Further research into the efficacy of non-surgical ablation techniques for patients with Bertolotti's Syndrome is warranted.

\section{CONCLUSION}

Bertolotti's Syndrome is a back-pain syndrome caused by a congenital defect of the last vertebra of the lumbar spine leading to the articulation, pseudo articulation, or full fusion of the transverse process to the sacrum the ilium. Bertolotti's Syndrome, which is present in approximately $7 \%$ of chronic lower back pain patients, is defined as a pain syndrome in conjunction with the LSTV. There are many treatment options for Bertolotti's Syndrome. However, a lack of research into the Syndrome hinders evidence supporting one specific therapy.

Outlined in Jancuska et al. is a guideline for the stepbased therapy for Bertolotti's Syndrome. ${ }^{10}$ It is essential to start with conservative methods, including activity modification, physiotherapy, steroid injections, and anesthetic injections. Failure of these modalities signals for a progres- 
sion towards interventional and or surgical management. Surgical methods, which have proved only slightly superior to non-surgical management, can differ depending on the preoperative degeneration of the spine. Resection and posterolateral fusion are both equally effective methods. Still, they both hold general surgical risks and the risk of revision surgery and further degeneration of the adjacent level. Radiofrequency ablation in any form (continuous RFA, pulsed RFA, cold RFA, or bipolar RFA) may provide an effective treatment method for patients with Bertolotti's Syndrome. Ablation can address the nociceptive and mechanical pain caused by Bertolotti's Syndrome, but there is currently a lack of research into RFA treatment for the variable presen- tations of LSTV. It is important that when patients present with back pain and LSTV, they are approached individually.

\section{DECLARATIONS OF INTEREST}

We declare no competing interests

CONFLICT OF INTEREST

None

Submitted: June 11, 2021 EST, Accepted: June 17, 2021 EST 


\section{REFERENCES}

1. Jain A, Agarwal A, Jain S, Shamshery C. Bertolotti syndrome: A diagnostic and management dilemma for pain physicians. Korean J Pain.

2013;26(4):368-373. doi:10.3344/kjp.2013.26.4.368

2. Alonzo F, Cobar A, Cahueque M, Prieto JA. Bertolotti's syndrome: An underdiagnosed cause for lower back pain. J Surg Case Reports. 2018;2018(10). d oi:10.1093/jscr/rjy276

3. Peterson CK, Bolton J, Hsu W, Wood A. A crosssectional study comparing pain and disability levels in patients with low back pain with and without transitional lumbosacral vertebrae. J Manipulative Physiol Ther. 2005;28(8):570-574. doi:10.1016/i.jmp t.2005.08.011

4. Fairbank JCT, Pynsent PB. The Oswestry Disability Index. Spine. 2000;25(22):2940-2452. doi:10.1097/000 07632-200011150-00017

5. Smeets R, Köke A, Lin CW, Ferreira M, Demoulin C. Measures of function in low back pain/disorders: Low Back Pain Rating Scale (LBPRS), Oswestry Disability Index (ODI), Progressive Isoinertial Lifting Evaluation (PILE), Quebec Back Pain Disability Scale (QBPDS), and Roland-Morris Disability Questionnaire. Arthritis Care Res. 2011;63(S11):S158-S173. doi:10.1002/acr.20 $\underline{542}$

6. Urits I, Burshtein A, Sharma M, et al. Low Back Pain, a Comprehensive Review: Pathophysiology, Diagnosis, and Treatment. Curr Pain Headache Rep. 2019;23(3):23. doi:10.1007/s11916-019-0757-1

7. Adams R, Herrera-Nicol S, Jenkins A III. Surgical Treatment of a Rare Presentation of Bertolotti's Syndrome from Castellvi Type IV Lumbosacral Transitional Vertebra: Case Report and Review of the Literature. J Neurol Surg Rep. 2018;79(03):e70-e74. do $\mathrm{i}: 10.1055 / \mathrm{s}-0038-1667172$

8. Manmohan S, Dzulkarnain A, Nor Azlin ZA, Fazir M. Bertolotti's Syndrome: A commonly missed cause of back pain in young patients. Malaysian Fam physician Off J Acad Fam Physicians Malaysia. 2015;10(2):55-58.

9. Castellvi AE, Goldstein LA, Chan DPK. Lumbosacral transitional vertebrae and their relationship with lumbar extradural defects. Spine. 1984;9(5):493-495. doi:10.1097/00007632-198407000-00014

10. Jancuska JM, Spivak JM, Bendo JA. A Review of Symptomatic Lumbosacral Transitional Vertebrae: Bertolotti's Syndrome. Int J Spine Surg. 2015;9(42):42. doi:10.14444/2042
11. Golubovsky JL, Colbrunn RW, Klatte RS, et al. Development of a novel in vitro cadaveric model for analysis of biomechanics and surgical treatment of Bertolotti syndrome. Spine J. 2020;20(4):638-656. do i:10.1016/i.spinee.2019.10.011

12. Bertolotti M. Contributo alla conoscenza dei vizi differenzazione regionle del rachid con speciale riguardo all'assimilazione sacrale edlla v lombare. $L a$ Radiolgoi Medica. 1917;4(4):113-144.

13. Paik NC, Lim CS, Jang HS. Numeric and morphological verification of lumbosacral segments in 8280 consecutive patients. Spine.

2013;38(10):E573-E578. doi:10.1097/brs.0b013e31828 $\underline{\mathrm{b} 7195}$

14. Otani K, Konno S, Kikuchi S. Lumbosacral transitional vertebrae and nerve-root symptoms. J Bone Jt Surg. 2001;83-B(8):1137-1140. doi:10.1302/03 01-620x.83b8.0831137

15. Vergauwen S, Parizel PM, Van Breusegem L, et al. Distribution and incidence of degenerative spine changes in patients with a lumbo-sacral transitional vertebra. Eur Spine J. 1997;6(3):168-172. doi:10.1007/ bf01301431

16. Hanhivaara J, Määttä JH, Niinimäki J, Nevalainen MT. Lumbosacral transitional vertebrae are associated with lumbar degeneration: Retrospective evaluation of 3855 consecutive abdominal CT scans. Eur Radiol. 2020;30(6):3409-3416. doi:10.1007/s0033 0-020-06691-2

17. Kanna RM, Shetty AP, Rajasekaran S. Predictors of Successful Outcomes of Selective Nerve Root Blocks for Acute Lumbar Disc Herniation. Glob Spine J. 2019;9(5):473-479. doi:10.1177/2192568218800050

18. Illeez OG, Atıcı A, Ulger EB, Kulcu DG, Ozkan FU, Aktas I. The transitional vertebra and sacroiliac joint dysfunction association. Eur Spine J. 2018;27(1):187-193. doi:10.1007/s00586-016-4879-4

19. Bron JL, Van Royen BJ, Wuisman PIJM. The clinical significance of lumbosacral transitional anomalies. Acta Orthopaedica Belgica. 2007;73:687-695.

20. Hsieh CYJ, Vanderford JD, Moreau SR, Prong T. Lumbosacral transitional segments: Classification, prevalence, and effect on disk height. J Manipulative Physiol Ther. 2000;23(7):483-489. doi:10.1067/mmt.2 000.108817 
21. Uçar D, Uçar BY, Coşar Y, et al. Retrospective Cohort Study of the Prevalence of Lumbosacral Transitional Vertebra in a Wide and Well-Represented Population. Arthritis. 2013;2013(461425):1-5. doi:10.1 $155 / 2013 / 461425$

22. Frymoyer JW, Newberg A, Pope MH, Wilder DG, Clements J, MacPherson B. Spine radiographs in patients with low-back pain. An epidemiological study in men. J Bone Jt Surg - Ser A.

1984;66(7):1048-1055. doi:10.2106/00004623-198466 070-00011

23. Elster AD. Bertolotti's Syndrome revisited: Transitional vertebrae of the lumbar spine. Spine. 1989;14(12):1373-1377. doi:10.1097/00007632-19891 2000-00015

24. Mitra R, Carlisle M. Bertolotti's Syndrome: A case report. Pain Pract. 2009;9(2):152-154. doi:10.1111/j.1 533-2500.2008.00253.x

25. Brenner AK. Use of lumbosacral region manipulation and therapeutic exercises for a patient with a lumbosacral transitional vertebra and low back pain. J Orthop Sports Phys Ther. 2005;35(6):368-376. $\underline{\mathrm{d}}$ oi:10.2519/jospt.2005.35.6.368

26. Muir JM. Partial lumbosacral transitional vertebrae: 2 cases of unilateral sacralization. J Chiropr Med. 2012;11(2):77-83. doi:10.1016/j.jcm.2011.12.002

27. Patti A, Bianco A, Paoli A, et al. Effects of Pilates Exercise Programs in People With Chronic Low Back Pain. Medicine. 2015;94(4):e383. doi:10.1097/md.0000 000000000383

28. Zhang R, Cheng J. Interventional Management of Chronic Low Back Pain Associated with Bertolotti's Syndrome: Report of Case Series. Ann Clin Case Reports. 2017;2:1348.

29. Holm EK, Bünger C, Foldager CB. Symptomatic lumbosacral transitional vertebra: A review of the current literature and clinical outcomes following steroid injection or surgical intervention. Sicot-J. 2017;3:71. doi:10.1051/sicotj/2017055

30. Jönsson B, Strömqvist B, Egund N. Anomalous Lumbosacral Articulations and Low-Back Pain. Spine. 1989;14(8):831-834. doi:10.1097/00007632-19890800 0-00009

31. Santavirta S, Tallroth K, Ylinen P, Suoranta H. Surgical treatment of Bertolotti's syndrome. Arch Orthop Trauma Surg. 1993;112(2):82-87. doi:10.1007/ bf00420261
32. Glémarec J, Varin S, Cozic C, et al. Efficacy of local glucocorticoid after local anesthetic in low back pain with lumbosacral transitional vertebra: A randomized placebo-controlled double-blind trial. Jt Bone Spine. 2018;85(3):359-363. doi:10.1016/j.jbspin.2017.05.003

33. Marks RC, Thulbourne T. Infiltration of anomalous lumbosacral articulations: Steroid and anesthetic injections in 10 back-pain patients. Acta Orthop Scand. 1991;62(2):139-141. doi:10.3109/17453 $\underline{679108999242}$

34. Ju CI, Kim SW, Kim JG, Lee SM, Shin H, Lee HY. Decompressive L5 transverse processectomy for Bertolotti's Syndrome: A preliminary study. Pain Phys. 2017;20(6):E923-E932. doi:10.36076/ppi.20.5.e9 $\underline{23}$

35. Brault JS, Smith J, Currier BL. Partial lumbosacral transitional vertebra resection for contralateral facetogenic pain. Spine. 2001;26(2):226-229. doi:10.1 097/00007632-200101150-00020

36. Haefeli M, Elfering A. Pain assessment. Eur Spine J. 2006;15(S1):S17-S24. doi:10.1007/s00586-005-104 $\underline{4-\mathrm{X}}$

37. Alcántara-Bumbiedro S, Flórez-García MT, Echávarri-Pérez C, García-Pérez F. Oswestry low back pain disability questionnaire. Rehabilitación. 2006;40(3):150-158. doi:10.1016/s0048-7120(06)7488 $\underline{1-2}$

38. Abe E, Sato K, Shimada Y, Okada K, Yan K, Mizutani Y. Anterior decompression of foraminal stenosis below a lumbosacral transitional vertebra: A case report. Spine. 1997;22(7):823-826. doi:10.1097/0 0007632-199704010-00023

39. Byrd D, Mackey S. Pulsed radiofrequency for chronic pain. Curr Pain Headache Rep. 2008;12(1):37-41. doi:10.1007/s11916-008-0008-3

40. Yin W, Willard F, Carreiro J, Dreyfuss P. Sensory stimulation-guided sacroiliac joint radiofrequency neurotomy: Technique based on neuroanatomy of the dorsal sacral plexus. Spine. 2003;28(20):2419-2425. d oi:10.1097/01.brs.0000085360.03758.c3

41. Cheng J, Pope JE, Dalton JE, Cheng O, Bensitel A. Comparative outcomes of cooled versus traditional radiofrequency ablation of the lateral branches for sacroiliac joint pain. Clin J Pain. 2013;29(2):132-137. d oi:10.1097/ajp.0b013e3182490a17

42. Cheng J, Chen SL, Zimmerman N, Dalton JE, Lasalle $\mathrm{G}$, Rosenquist R. A new radiofrequency ablation procedure to treat sacroiliac joint pain. Pain Phys. 2016;19(8):603-615. doi:10.36076/ppj/2016.19.6 $\underline{03}$ 
43. Burnham R. Radiofrequency Sensory Ablation as a Treatment for Symptomatic Unilateral Lumbosacral Junction Pseudarticulation (Bertolotti's Syndrome): A Case Report. Pain Med. 2010;11(6):853-855. doi:10.11 11/j.1526-4637.2010.00869.x

44. De Almeida DB, Mattei TA, Sória MG, et al. Transitional lumbosacral vertebrae and low back pain: Diagnostic pitfalls and management of Bertolotti's syndrome. Arq Neuropsiquiatr. 2009;67(2a):268-272. doi:10.1590/s0004-282x200900 $\underline{0200018}$
45. Kanematsu R, Hanakita J, Takahashi T, Tomita Y, Minami M. Pulsed Radiofrequency Treatment for Bertolotti's Syndrome Presenting With Low Back Pain: Report of Four Cases. Pain Pract. 2020;20(5):568-570. doi:10.1111/papr.12877

46. Simopoulos TT, Malik AB, Sial KA, Elkersh M, Bajwa ZH. Radiofrequency lesioning of the L2 ramus communicans in managing discogenic low back pain. Pain Physician. 2005;8(1):61-65.

47. Saal JS, Saal JA. Management of chronic discogenic low back pain with a thermal intradiscal catheter: A preliminary report. Spine.

2000;25(3):382-388. doi:10.1097/00007632-20000201 $\underline{0-00021}$ 